\title{
Modulation of homogeneous space-time rainfall cascades to account for orographic influences
}

\author{
M. G. Badas, R. Deidda, and E. Piga \\ Dipartimento di Ingegneria del Territorio, Università di Cagliari, Piazza d'Armi, 09123 Cagliari, Italy \\ Received: 18 October 2005 - Revised: 2 February 2006 - Accepted: 2 February 2006 - Published: 6 June 2006
}

\begin{abstract}
The development of efficient space-time rainfall downscaling procedures is highly important for the implementation of a meteo-hydrological forecasting chain operating over small watersheds. Multifractal models based on homogeneous cascade have been successfully applied in literature to reproduce space-time rainfall events retrieved over ocean, where the hypothesis of spatial homogeneity can be reasonably accepted. The feasibility to apply this kind of models to rainfall fields occurring over a mountainous region, where spatial homogeneity may not hold, is herein investigated. This issue is examined through the analysis of rainfall data retrieved by the high temporal resolution rain gage network of the Sardinian Hydrological Survey. The proposed procedure involves the introduction of a modulating function which is superimposed to homogeneous and isotropic synthetic fields to take into account the spatial heterogeneity detected in observed precipitation events. Specifically the modulating function, which reproduces the differences in local mean values of the precipitation intensity probability distribution, has been linearly related to the terrain elevation of the analysed spatial domain. Comparisons performed between observed and synthetic data show how the proposed procedure preserves the observed rainfall fields features and how the introduction of the modulating function improves the reproduction of spatial heterogeneity in rainfall probability distributions.
\end{abstract}

\section{Introduction}

Severe precipitation events occurring over small watersheds are often caused by localized convective cells belonging to synoptic systems. In such cases basin response times can be too short to issue an alert on the basis of real time pre-

Correspondence to: R. Deidda

(rdeidda@unica.it) cipitation data retrieved by radar or rain gage telemeters. It is thus necessary to rely on meteorological forecast in order to predict precipitation fields and to evaluate their possible effects on ground. Nevertheless numerical weather prediction (NWP) models alone are unlikely to provide rainfall fields adequate for an efficient application of rainfall-runoff models to small basins. Specifically general circulation models (GCMs) resolution is larger than the required hydrological scales. Limited area models (LAMs) can be nested into GCMs in order to increase the resolution, but the coarseness of the observation network, which is used to build the meteorological analyses and to initialize GCMs, may deeply affect the subsequent LAM forecast. Specifically this problem may influence the localization and quantification of rainfall peaks in high resolution space-time LAMs domains. Therefore, before using them in a forecasting chain, it may be appropriate to reaggregate high resolution NWP forecasts to a coarser scale where rainfall predictions become reliable, as pointed out by Siccardi et al. (2005).

Thus the development of effective procedures for precipitation downscaling has a prominent role in forecasting hydrogeological risk for small watersheds. Starting from a rainfall event predicted over a large space-time scale by a NWP model, statistical downscaling allows generating several equally probable realizations of the same event at scales compatible with rainfall-runoff models. When embedded in a forecasting chain, downscaling procedures can thus be applied to account for small scales uncertainties in rainfall input and their coupling with rainfall-runoff models may help to provide a probabilistic scenario of the ground effects.

Apart from the uncertainty related to the gap of scales between meteorological and hydrological models many other factors may affect the reliability of a forecasting chain. The ensemble prediction system (EPS) that is currently running at the ECMWF (European Center for Medium range Weather Forecasting) allows accounting for meteorological model uncertainty due to the coarseness of the observation network

Published by Copernicus GmbH on behalf of the European Geosciences Union. 
and to the chaoticity of equations used in meteorological models (Buizza et al., 1999). As the last element of a forecasting chain, hydrological models are also affected by uncertainties that are mainly due to our limited knowledge of catchment subsurface properties (Beven, 2001). In this paper we focus the problem of rainfall downscaling with special regard to mountain regions, while for a discussion on the different sources of model uncertainties and their influence on a forecasting chain, the reader is referred to Ferraris et al. (2002) and Siccardi et al. (2005), as it goes beyond the aim of this work.

An important class of statistical models useful for rainfall downscaling is represented by multifractal models, which are able to reproduce rainfall variability observed at different space and time scales. Statistical properties of rainfall fields are usually characterized by means of multifractal theory applying self-similar or self-affine transformations. Some recent studies performed on radar rainfall dataset retrieved during the two international campaigns GATE (GARP, Global Atmospheric Research Program, Atlantic Tropical Experiment) and TOGA-COARE (Tropical Ocean Global Atmosphere Coupled Ocean-Atmosphere Response Experiment) gave evidence of a scale invariant behaviour with simple selfsimilar transformations (Deidda, 2000; Deidda et al., 2004).

Both GATE and TOGA-COARE precipitation fields were retrieved over the ocean without any physical obstacle which could bias the spatial distribution of rainfall probability in the analysed domain. On the contrary, when precipitation fields need to be generated in presence of orographic obstacles and barriers, it is convenient to analyse the effects of these constraints on rainfall fields and on local probability rainfall distributions. Actually mean and extreme precipitation values are known to vary with the terrain elevation. More generally local probability distribution of rainfall may be influenced by many morphological factors besides the altitude: slope and shape of orographic relieves, as well as their exposition with respect to the direction of the perturbation. However it may be rather difficult to deduct the influence of each of these factors from the analysis of point rainfall data retrieved by rain gages.

The opportunity of introducing a heterogeneous component when modelling synthetic rainfall fields over land was investigated by Harris et al. (1996), Jothityangkoon et al. (2000), Purdy et al. (2001), Pathirana and Herath (2002). Specifically Jothityangkoon et al. (2000) and Pathirana and Herath (2002) analysed a $400 \mathrm{~km} \times 400 \mathrm{~km}$ area in southwestern Australia and a $128 \mathrm{~km} \times 128 \mathrm{~km}$ region centered in the Japanese archipelago, respectively. In order to reproduce observed spatial heterogeneity they multiplied a homogeneous spatial random cascade by a deterministic factor depending on the spatial location without linking it to local features. Analyzing a transect along the Southern Alps of New Zealand, Harris et al. (1996) and Purdy et al. (2001) found a dependence of scaling parameters on orography and rain features. More recently Deidda et al. (2006) investigated the presence of rainfall spatial heterogeneity induced by orography on rainfall fields retrieved over Brazil by radars (TRMMLBA, Tropical Rainfall Measurement Mission - Large Scale Biosphere Atmosphere Experiment). Nevertheless this analysis did not highlight the presence of any spatial heterogeneity, probably because of the absence of orographic barriers that could significantly force upward air movements.

The issue of rainfall downscaling over a mountainous region was examined by Badas et al. (2005) and is deepened in the present paper. We propose a procedure that allows the reproduction of spatial heterogeneity detected in observed data, provided that they are not affected by local variations in the multifractal behaviour. It consists in the modulation of spacetime homogeneous cascades by means of a simple modulating function adequately calibrated. Specifically the analysis of the orographic influence on precipitation observed by the high temporal resolution rain gage network of the Sardinian Hydrological Survey is discussed. The examined data were collected in the period from 1986 to 1996 by 235 rain gage stations (Fig. 1), which recorded on a memory chip the instants when $0.2 \mathrm{~mm}$ of rainfall were cumulated.

The paper consists of the following sections. In Sect. 2 we recall some important features of multifractal downscaling models with special regard to the STRAIN model (Deidda, 2000) which has been used in the present study. In Sect. 3 we present a methodology introducing spatial heterogeneity in the rainfall disaggregation by means of a modulating function. This function is determined in Sect. 4 on the basis of some preliminary analyses based on both rain gage observations and average rainfall computed on the cells displayed in Fig. 1. In Sect. 5 the scale-invariance analysis performed on space-time domains is described, while in Sect. 6 the results obtained for numerical simulations are discussed. Finally in Sect. 7 the conclusions of the work are drawn.

\section{Rainfall downscaling by means of multifractal theory}

Multifractal theory led to the development of an important class of statistical models useful for rainfall downscaling. Mainly due to empirical evidences, the application of multifractal models for rainfall downscaling, which allows to reproduce hierarchical structures of rainfall fields shown by Austin and Houze (1972), is widespread in literature (Lovejoy and Mandelbrot, 1985; Schertzer and Lovejoy, 1987; Gupta and Waymire, 1993; Over and Gupta, 1996; Perica and Foufoula-Georgiou, 1996; Deidda, 2000, among the others).

What follows is a brief framework for the analysis of scaleinvariant space-time precipitation fields. Let $i(x, y, t)$ represent the rainfall intensity at location $(x, y)$ and time $t$, the rainfall volume corresponding to a generic space-time region $\lambda \times \lambda \times \tau$ located in $x_{i}, y_{j}, t_{k}$ can be computed as follows:

$\mu_{i, j, k}(\lambda)=\int_{x_{i}}^{x_{i}+\lambda} d x \int_{y_{j}}^{y_{j}+\lambda} d y \int_{t_{k}}^{t_{k}+\lambda / U} d t i(x, y, t)$ 


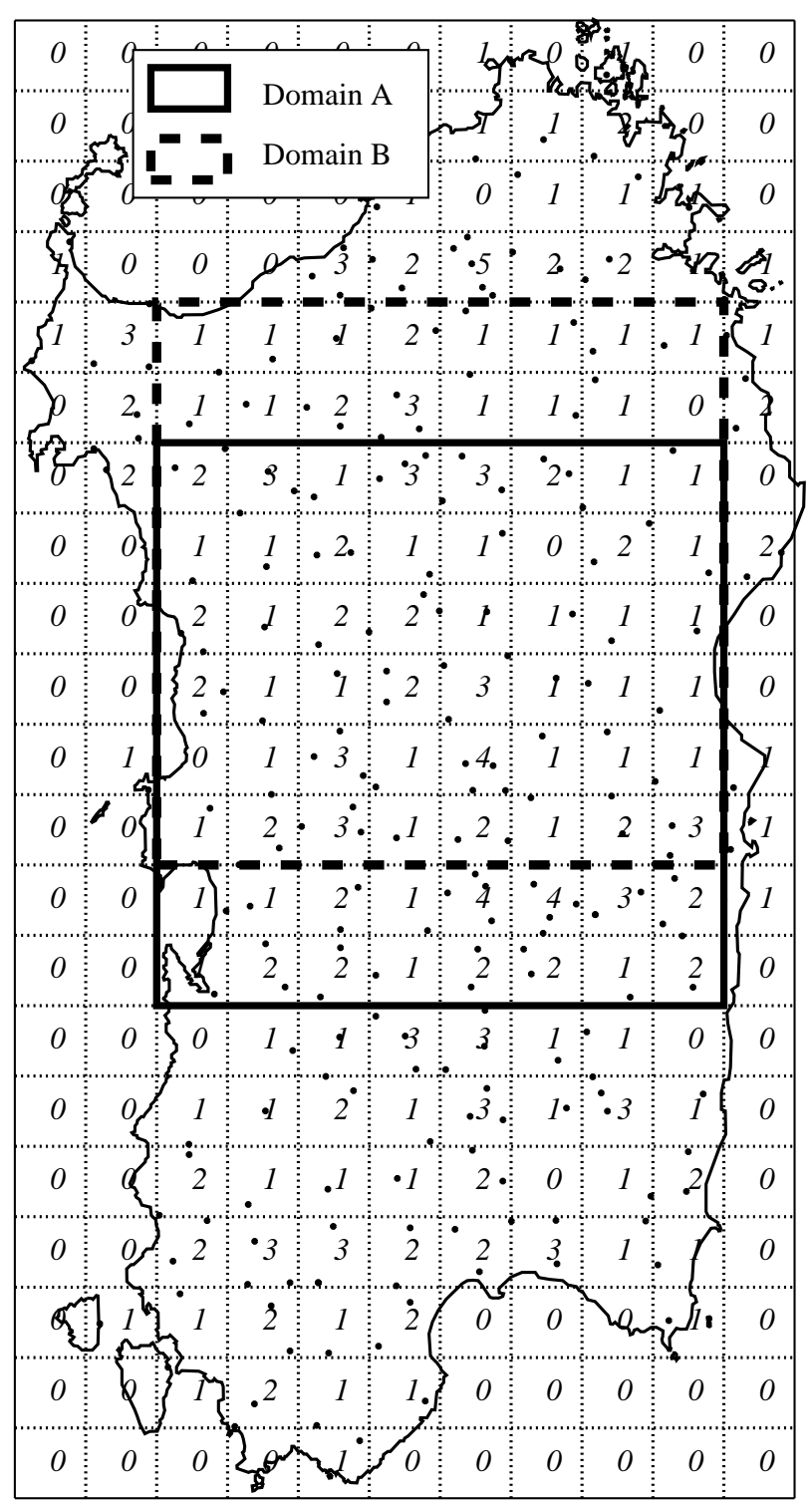

Fig. 1. The regular grid with $13 \mathrm{~km}$ resolution used for the analysis is superimposed to the sardinian coast. The number of rain gages belonging to each cell is reported inside the cell, while points indicate the station positions. A and B domains correspond to the regions where the space-time sequences analysed in Sects. 4 and 5 were extracted.

In Eq. (1) the integration time scale $\tau$ is related to the spatial scale $\lambda$ by the relationship $\tau=\lambda / U$, where $U$ may be or not a function of $\lambda$, as it will clearly appear further on. With reference to Fig. 2 we can evaluate rainfall volumes $\mu$ at different space-time domains ranging from the large scale $L \times L \times T$ resolved by meteorological models to the smaller scales $\lambda_{0} \times \lambda_{0} \times \tau_{0}$ compatible with the watershed extension. Given the rainfall volume over the large scales $L \times L \times T$ a multifractal downscaling model should be able to determine the probability distribution function of rainfall volumes over

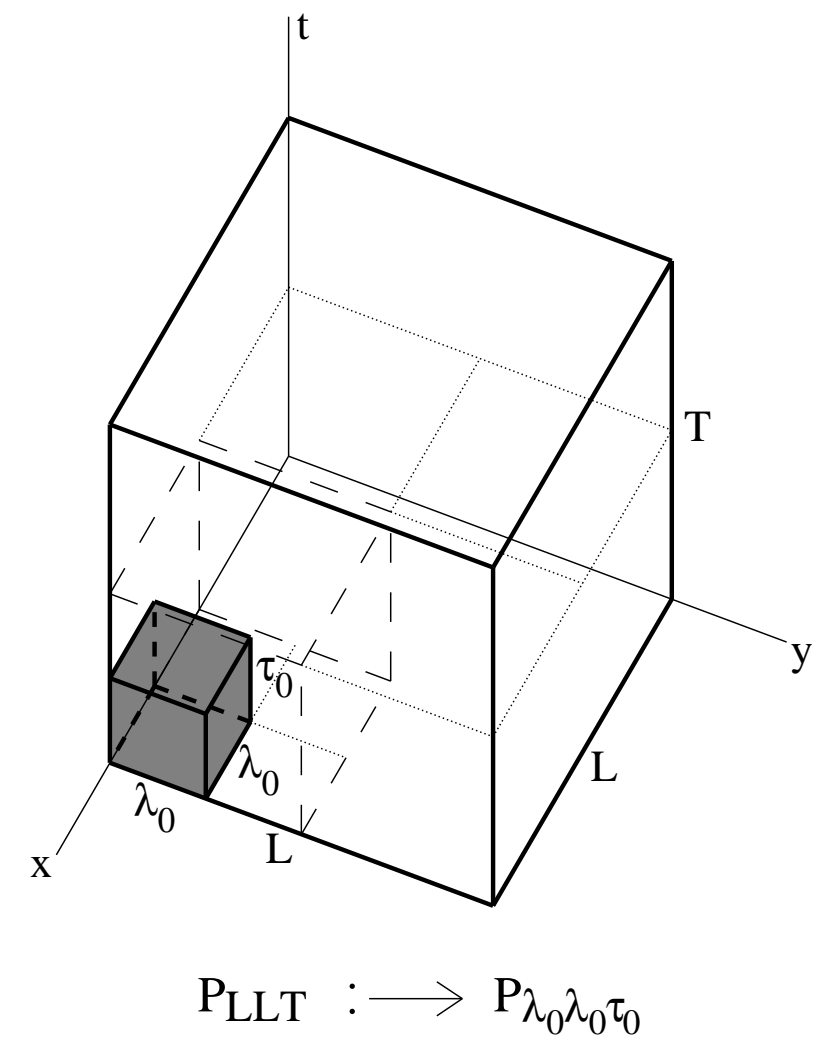

Fig. 2. A schematization of a downscaling problem: a rainfall measure is given over a region $L \times L \times T$ (e.g., the grid resolution of a NWP model), we want to determine the probability distribution of rainfall intensity over any smaller region up to the smallest scales $\lambda_{0} \times \lambda_{0} \times \tau_{0}$.

smaller scales $\lambda_{0} \times \lambda_{0} \times \tau_{0}$, preserving some kind of scale invariance rules.

Scale invariant properties of rainfall fields have to be investigated all over the selected space-time scale range in order to assess whether a multifractal model can be applied. In the simplest scenario of self-similarity (or scale isotropy) a scale-independent velocity parameter $U$ can be introduced to transfer the statistical properties observed at space scales $\lambda$ to coherent time scales $\tau=\lambda / U$ and vice versa. Thus scale invariant properties can be studied on space-time domains derived by isotropic transformations which are characterized by the same branching number $b_{s}$ for both space and time: $x \rightarrow x / b_{s}, y \rightarrow y / b_{s}, t \rightarrow t / b_{s}$.

Once the scale-invariant velocity parameter $U$ needed to relate coherent space and time scales is determined, the following $q$-order partition functions $S_{q}(\lambda)$ can be computed at different scales $\lambda$ :

$S_{q}(\lambda)=\frac{1}{N(\lambda)^{2} N(\tau)} \sum_{i=1}^{N(\lambda)} \sum_{j=1}^{N(\lambda)} \sum_{k=1}^{N(\tau)} \mu_{i, j, k}(\lambda)^{q}$ 
where $N(\lambda)^{2} N(\tau)$ is the number of subregions $\lambda \times \lambda \times \tau$ of the $\lambda$-partition, being $N(\lambda)$ and $N(\tau)=N(\lambda)$ the number of subgrid cells in each direction of space and time respectively. In terms of partition functions, scale-invariance laws can be written as follows:

$S_{q}(\lambda) \sim \lambda^{\zeta(q)}$

The non linearity of the $\zeta(q)$ exponents reveals the multifractality of the measure $\mu$, on the contrary if $\zeta(q)$ is a linear function of $q$ the field is monofractal.

For the sake of generality and to perserve the formalism used by other authors, we briefly review how a scale invariant analysis can be performed for fields displaying selfaffinity (i.e. space-time anisotropy). Actually the above definitions given for self-similar measures can be easily generalized to a self-affine framework thanks to anisotropic spacetime transformations: $x \rightarrow x / b_{s}, y \rightarrow y / b_{s}, t \rightarrow t / b_{t}$, where the branching number $b_{s}$ in space now differs from the one $b_{t}$ in time. However space and time branching number are not independent: according to the "Generalized Scale Invariance" (G.S.I.) (Lovejoy and Schertzer, 1985; Schertzer and Lovejoy, 1985) they are related by $b_{t}=b_{s}^{(1-H)}$; while using the "dynamic scaling" formalism (Kardar et al., 1986; Czirok et al., 1993; Venugopal et al., 1999a,b) the previous relationship becomes $b_{t}=b_{s}^{z}$. The "scaling anisotropy exponent" $H$ in the G.S.I. formalism, or equivalently the "dynamic scaling exponent" $z$ using the second approach, characterize the degree of scale anisotropy; a strict connection holds between the two parameters: $z=1-H$. In case of selfaffinity, space and time scales are made homogeneous by a scale-dependent velocity parameter $U_{\lambda} \sim \lambda^{H}$, while in the trivial case of self-similarity $(H=0$ or $z=1) U$ becomes a constant value. Although the self-affine approach may appear to be attractive for its generality, there are several analyses on rainfall scale-invariance indicating that the simpler self-similar assumption allows to satisfactory explain rainfall multifractality. For more details the reader can refer to Deidda (2000) and Deidda et al. (2004).

If space-time rainfall fields display scale invariant properties (Eq. 3) they can be modelled by means of stochastic multiplicative cascades, which were first introduced in fluid dynamics in order to reproduce the turbulent energy transfer mechanism. Among the models for rainfall simulation described in literature, the STRAIN model (Deidda et al., 1999; Deidda, 2000) has been here chosen to represent the analysed rain fields. It is based on a log-Poisson generator $\eta=e^{A} \beta^{y}$, where $y$ is a Poisson-distributed random variable with mean $c$. The model is parsimonious, indeed only $c$ and $\beta$ are free parameters, since the "mean conservation" condition $\bar{\eta}=1$ allows eliminating the third one immediately: $A=c(1-\beta)$. Besides many other properties (Dubrulle, 1994; She and Leveque, 1994; She and Waymire, 1995), the logPoisson generator satisfies the moments convergence condition for a wide parameters range (Deidda et al., 2004) which includes the bulk of the estimates obtained for the data here analysed. We recall the theoretical expectation for the multifractal exponents $\zeta(q)$ of the power law (Eq. 3), which allows the calibration of the model parameters:

$\zeta(q)=d q-c \frac{q(1-\beta)-\left(1-\beta^{q}\right)}{\ln 2}$

where $d$ is the dimension of the region $S$ supporting the measure $\mu$ (in our space-time downscaling problem $d=3$ ) while a common binary branching number $\left(b_{s}=b_{t}=2\right)$ is assumed in each direction of the region $S$. For a deeper insight on the STRAIN model the reader is referred to the above cited bibliography.

Parameters of multifractal cascade models are usually estimated from sample multifractal moments $\zeta(q)$, once their theoretical behaviour has been derived from model generator structure. However attention has to be paid when choosing the $q$ values in order to calibrate the model. Actually estimates with low $q$ values are not reliable because they emphasize low frequency noise and discretization effects, especially in case of tipping bucket rain data. On the other side, high $q$ values magnify the contribution of intense events, which can be a few according to the sample size. If $q$ exceeds a certain value sample multifractal exponents behaviour becomes linear. This effect has often been ascribed to the sample finiteness or to the moments divergence (Schertzer and Lovejoy, 1987; Mandelbrot, 1990); however Lashermes et al. (2004) proved this feature to derive from the process nature rather than being tied to multifractal exponents estimation procedures.

Multifractal exponents $\zeta(q)$ in the scale-invariant law (Eq. 3) can be also related to the modified cumulant generating function $\chi_{b}(q)$ (Mandelbrot, 1974; Kahane and Peyriere, 1976), often referred to as MKP function :

$\zeta(q)=d\left[1-\chi_{b}(q)\right]$

where $b=b_{s}^{d}$ for self-similar multifractals embedded on the region $S \subset R^{d}$ ( $d=3$ for space-time fields), while the MKP function is defined by:

$\chi_{b}(q)=\log _{b} \overline{\eta^{q}}-(q-1)$

Kahane and Peyriere (1976) stated the conditions on $\chi_{b}(q)$ assuring nondegeneracy of $\mu_{\infty}$ (i.e. the measure of a fully developed cascade also referred to as a "dressed measure"), the convergence of its moment, and deduced the estimation of its support dimension. More recently Ossiander and Waymire $(2000,2002)$ stated a theorem for consistency, which asymptotically establishes the conditions needed from data sampled at a prescribed fine scale resolution to consistently determine the distribution of the cascade generator. All these constraints have to be taken into account when calibrating a multifractal model on the basis of sample data. 


\section{Introduction of local heterogeneity in synthetic rain- fall fields}

What we propose in this paper is a simple methodology allowing to investigate spatial heterogeneity in rainfall fields and to introduce corresponding constraints in downscaling models. Let us consider the domain $L \times L \times T$ of Fig. 2 whose mean rainfall intensity $I$ for each precipitation event may be computed as follows:

$I=\frac{1}{L L T} \int_{0}^{L} \int_{0}^{L} \int_{0}^{T} i(x, y, t) d x d y d t$

The ratio between mean rainfall intensity of each cell $(x, y)$ and the mean rainfall $I$ in the selected domain can be assumed as a local modulating function expressed by:

$\xi(x, y)=\frac{\frac{1}{T} \int_{0}^{T} i(x, y, t) d t}{I}$

The function $\xi(x, y)$ computed on a single rainstorm shows high variability not only due to the effect of orography but also to the specific feature of each precipitation event. Sample variability depending on the single rainstorm features can be filtered by averaging $\xi(x, y)$ estimates of many events. Thus the assessment of possible orographic constraints in the studied region should be pursued on the basis of the spatial distribution of sample mean $\bar{\xi}(x, y)$. In a region where the orography does not exert any kind of conditioning on rainfall patterns we should expect rainfall to be spatially homogeneous on average, leading the average modulating function $\bar{\xi}(x, y)$ to be a constant value (equal to one), whatever the location of the point $(x, y)$ is. On the contrary, if the pattern of the modulating function $\bar{\xi}(x, y)$ highlights a dependence on localization, this represents a territorial heterogeneity which has to be reproduced also in the synthetic fields. This kind of spatial heterogeneity can be modelled multiplying a homogeneous random cascade $i_{0}(x, y, t)$ by the modulating function $\bar{\xi}(x, y)$ :

$i(x, y, t)=\bar{\xi}(x, y) i_{0}(x, y, t)$

where $i(x, y, t)$ represent the resulting heterogeneous rainfall field.

This approach is similar to the one adopted by Jothityangkoon et al. (2000) and Pathirana and Herath (2002), who modulated homogeneous spatial random cascades by means of spatial patterns obtained from long term accumulation rain fields.

\section{Estimate of the modulating function}

The proposed procedure was calibrated, applied and tested to rainfall data retrieved by the high temporal resolution rain gage network of the Sardinian Hydrological Survey and illustrated in Sect. 1. In order to analyse scale invariant properties and multifractal behaviour of space-time rain fields, raw data were averaged on the grid displayed in Fig. 1, where points indicate rain gage stations location. The adopted grid spatial step $\lambda_{0}=13 \mathrm{~km}$ was obtained dividing the island width $L=104 \mathrm{~km}$ by an integer power of two (corresponding to the branching number $b_{s}=2$ ), with at least one rain gage in most of the cells. The comparison of self-correlation functions in space and time allowed to estimate the velocity parameter $U=17.33 \mathrm{~km} / \mathrm{h}$ needed for space-time transformations in the adopted self-similar framework. Therefore the smallest time resolution is $\tau_{0}=\lambda_{0} / U=45 \mathrm{~min}$, while the examined event duration is $T=L / U=6 \mathrm{~h}$. Figure 1 shows the grid and the spatial domains used both for the space-time scale invariant analysis and the downscaling process.

The research of a modulating function has been preliminary performed on raw rain gage data and then on data gridded on the cells of the grid described above. Both the analyses were made on intense precipitation events with a duration $T$ equal to $6 \mathrm{~h}$; results concordantly indicate the opportunity to modulate the rainfall downscaling process at least according to the terrain elevation.

\subsection{Analysis on rain gage data}

The analysis on rain gage data was performed on independent events selected on a regional scale. The regional mean intensity $I_{\theta}^{(p)}$ on a time interval of duration $T=6 \mathrm{~h}$ was preliminary computed for all the potential events beginning at a generic time $\theta$ :

$I_{\theta}^{(p)}=\frac{1}{N_{p}} \sum_{k=1}^{N_{p}} \frac{1}{T} \int_{\theta}^{\theta+T} i_{k}(t) d t$

where $N_{p}$ is the number of active recording rain gages in the whole region from $\theta$ to $\theta+T$, and $i_{k}(t)$ is rainfall intensity retrieved by the $k$-th rain gage at time $t$.

The most intense 794 independent events were then selected, with a resulting average intensity $I_{\theta}^{(p)}$ ranging from $0.2 \mathrm{~mm} / \mathrm{h}$ and $4.5 \mathrm{~mm} / \mathrm{h}$. For each event starting at time $\theta$ and for each rain gage $k$ the function $\xi_{\theta}(k)=\left(\frac{1}{T} \int_{\theta}^{\theta+T} i_{k}(t) d t\right) / I_{\theta}^{(p)}$ was computed. Sample variability was filtered by averaging for each rain gage $k$ the function $\xi_{\theta}(k)$ over all the selected events. The analysis of the results showed a spatial variability of the average $\bar{\xi}(k)$ function which can be partially explained considering the rain gage elevation $z(k)$. As illustrated in Fig. 3 the comparison between modulating functions $\bar{\xi}(k)$ and rain gage elevation $z(k)$ shows a behaviour which can be linearly interpreted, despite a noticeable dispersion of the representative points. The regression line slope $\alpha$ computed on sample points and shown in Fig. 3 is equal to $0.61 / 1000 \mathrm{~m}^{-1}$.

\subsection{Analysis on a regular grid}

With the aim to obtain a feasible relationship for the analysis and the simulation of space-time rainfall events, the $\xi$ function was also examined for rainfall data averaged on the same 


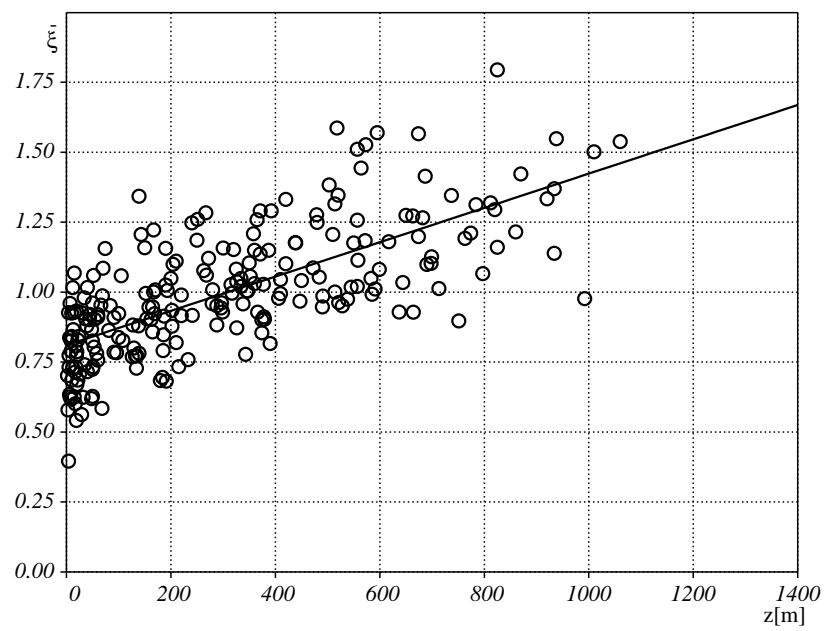

Fig. 3. Values of the modulating function $\bar{\xi}(k)$ versus rain gage elevation $z(k)$ and corresponding regression line.

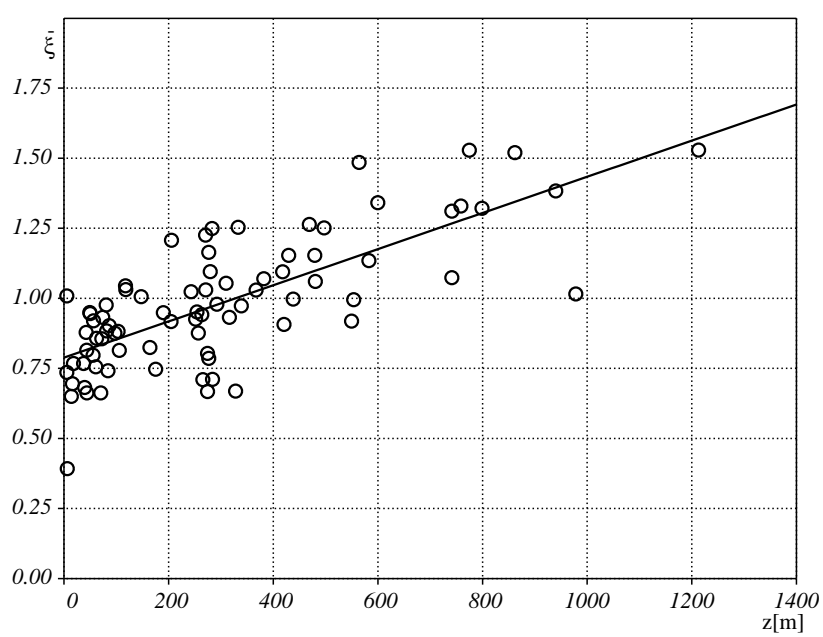

Fig. 4. Modulating function values $\bar{\xi}(x, y)$ versus mean grid cell elevation $z(x, y)$ and corresponding regression line.

regular grid used in the downscaling process and already displayed in Fig. 1. The regional mean intensity $I_{\theta}^{(g)}$ on $T=6 \mathrm{~h}$ and on the whole spatial grid was in this case computed as:

$I_{\theta}^{(g)}=\frac{1}{N_{g}} \sum_{k=1}^{N_{g}} \frac{1}{T} \int_{\theta}^{\theta+T} i_{k}(t) d t$

where $N_{g}$ is the number of grid cells with at least one rain gage working during the event, $\theta$ is the beginning of the event, while $i_{k}(t)$ is the average rainfall intensity of rain gages within the $k$-th grid cell.

The most intense 806 events were selected with a resulting mean intensity $I_{\theta}^{(g)}$ ranging from $0.2 \mathrm{~mm} / \mathrm{h}$ and $4.5 \mathrm{~mm} / \mathrm{h}$. Such as for the case of raw rain gage data, for each grid cell $(x, y)$ the mean value $\bar{\xi}(x, y)$ on all the events was com-

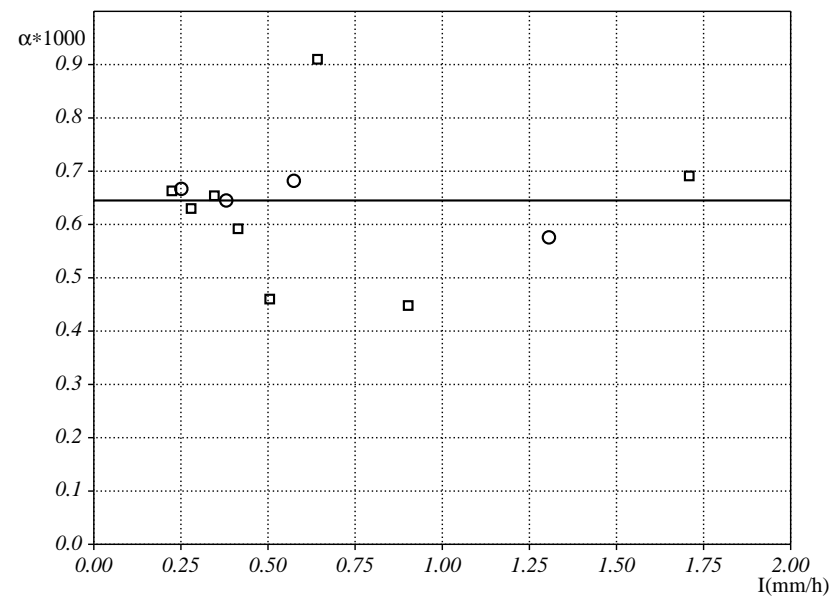

Fig. 5. Slopes $\alpha$ obtained dividing the selected events into four groups (circles) and eight groups (squares) versus mean group rain intensity $I$. Solid line represents mean $\alpha$ value computed on all the 806 events.

puted. Figure 4 shows the behaviour of the modulating function $\bar{\xi}(x, y)$ with the average elevation $z(x, y)$ of the cell: the regression line slope $\alpha=0.65 / 1000 \mathrm{~m}^{-1}$. As expected, this value is similar to the one obtained for single rain gage data since gridded data have been obtained from the same rain gage network that, being quite dense, does not introduce significant biases in $\xi$ estimates.

With the aim of verifying the stability of $\alpha$ estimates, the dataset containing 806 sequences has been divided according to mean rainfall intensity $I_{\theta}^{(g)}$ into four and then eight groups having the same number of elements. Mean values $\bar{\xi}(x, y)$ and slopes $\alpha$ of the regression lines (in the $\xi-z$ plane) have been computed for each group. The $\alpha$ values are plotted in Fig. 5 versus the mean rainfall intensity of the group. Figure 5 shows that these values do not display any trend with intensity, $\alpha$ has been thus considered constant and equal to its mean value $0.65 / 1000 \mathrm{~m}^{-1}$, which was estimated on the 806 events.

In order to represent the behaviour of the modulating function $\bar{\xi}(x, y)$ with the average cell elevation $z(x, y)$ the following equation was thus assumed:

$\bar{\xi}(x, y)=\alpha z(x, y)+b$

where the intercept $b=1-\alpha<z>_{(x, y)}$ is easily obtained from the relationships (7) and (8), while $<\cdot>_{(x, y)}$ is the spatial average on the selected domain. Thus it is possible to define the functional relationship between $\bar{\xi}$ and the terrain elevation for different spatial domains, assuring the conservation of the large scale rainfall volume. 


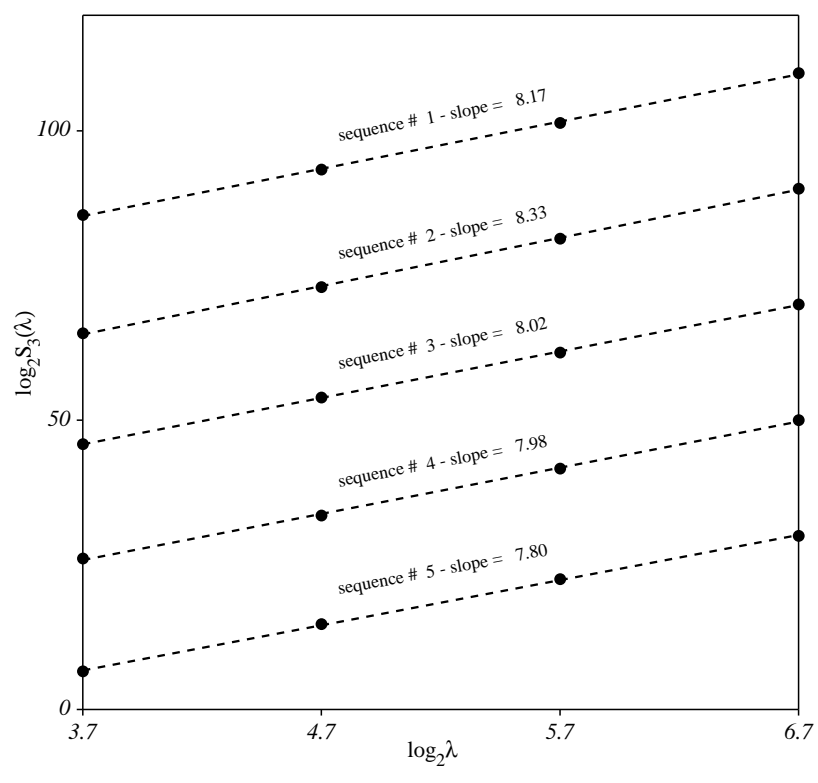

Fig. 6. Partition functions of the third order $S_{3}(\lambda)$ computed on spatial scales ranging from $\lambda_{0}=13 \mathrm{~km}$ to $L=104 \mathrm{~km}$ (and corresponding time scales from $\tau_{0}=45 \mathrm{~min}$ a $T=6 \mathrm{~h}$ ) for the 5 most intense sequences. The partition functions are plotted in arbitrary units in order to display different sequences results in the same graph. For each field the multifractal exponent $\zeta(3)$, slope of the regression line, is indicated.

\section{Scale invariant properties on rainfall events extracted on a regular grid}

Although the computation of the modulating function $\xi$ has been performed on events which could contain some missing rain data, for the following multifractal analyses the conditions imposed for events selection were stricter. Specifically we accepted for each event a maximum of $20 \%$ missing data; in order to perform scale invariance and multifractal analyses these precipitation measures were estimated averaging the rainfall intensities retrieved over the adjacent eight grid cells in the same time interval $\tau_{0}=45 \mathrm{~min}$. The stricter condition imposed for missing data allowed us to select only 138 space-time events that occurred over the A and B domains displayed in Fig. 1. These sequences were retrieved over a $L \times L \times T$ domain with dimension $L=104 \mathrm{~km}$ in space and $T=6 \mathrm{~h}$ in time; their resolution is $\lambda_{0}=13 \mathrm{~km}$ and $\tau_{0}=45 \mathrm{~min}$, while the mean rain intensities $I$ range from 0.2 to $3.5 \mathrm{~mm} / \mathrm{h}$. For the following analyses, the selected sequences were made spatially homogeneous dividing rainfall intensity of each cell $(x, y)$ by the corresponding modulating function value $\bar{\xi}(x, y)$ defined by the Eq. (12) for the respective domain.

The selected space-time sequences were found to be selfsimilar on the average, with a scale independent velocity parameter $U=17.33 \mathrm{~km} / \mathrm{h}$ relating coherent space and time scales. We computed for each sequence the parti-

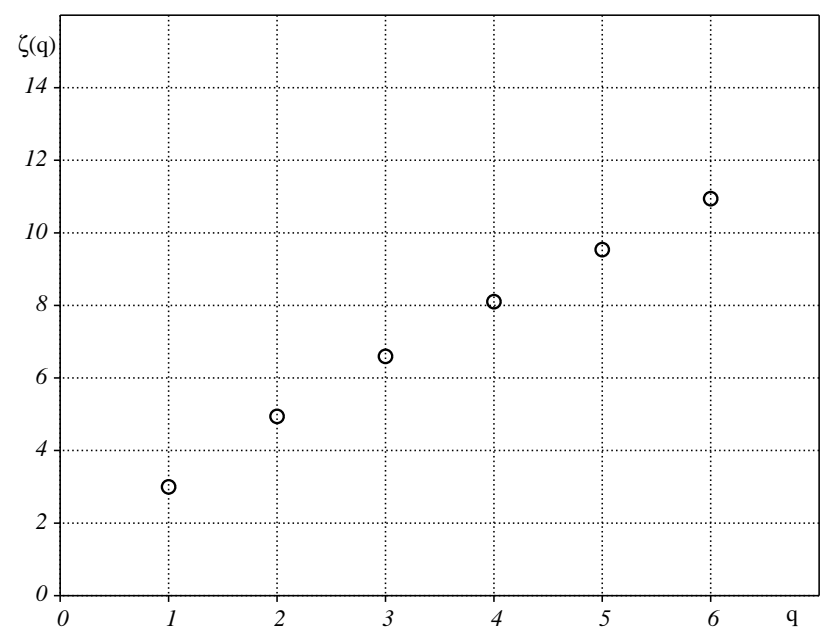

Fig. 7. Sample multifractal exponents $\zeta(q)$ computed for one of the selected sequences are plotted versus the moment order $q$ and display the typical multifractal non-linear behaviour.

tion functions $S_{q}(\lambda)$ defined in Eq. (2) on space scales $\lambda$ from $\lambda_{0}=13 \mathrm{~km}$ to $L=104 \mathrm{~km}$ and corresponding time scales $\tau$ from $\tau_{0}=\lambda_{0} / U=45 \mathrm{~min}$ to $T=L / U=6 \mathrm{~h}$. The partition functions $S_{q}(\lambda)$, calculated for moments $q$ from 1 to 6 , showed a good correspondence to the scaling law (Eq. 3) for all the space and time scales considered. As an example in Fig. 6 the third order partition functions $S_{3}(\lambda)$ of the most intense 5 sequences are plotted versus $\lambda$. Figure 6 clearly shows that sample points are well interpreted by scaling laws (Eq. 3) which correspond to linear trends in the log-log plane. The regression line slopes, given in Fig. 6, provide estimates of the $\zeta(q)$ multifractal exponents. All the analysed events show the non-linear behaviour typical of multifractal fields when plotting $\zeta(q)$ multifractal exponents versus $q$, as displayed in Fig. 7 for one event.

The behaviour of the multifractal exponents $\zeta(q)$ was interpreted by the STRAIN cascade model briefly reviewed in Sect. 2. Showing a behaviour similar to the one already found for the GATE and TOGA-COARE dataset, $\beta$ estimates do not display a great variability among the different sequences, therefore $\beta$ was considered as constant and equal to $e^{-1}$. The $c$ parameter estimates, bounded to the constant $\beta$ value, showed (despite a high variability) a decreasing trend with increasing mean intensities. This behaviour, displayed in Fig. 8 (top), was interpreted with the following equation:

$c(I)=a \exp (-\gamma I)+c_{\infty}$

where $a=1.3679, \gamma=1.4792, c_{\infty}=0.8257$.

The form of the Eq. (13) is the same of the one used to interpret the $c$ behaviour for GATE, TOGA-COARE, and TRMM-LBA sequences (Deidda, 2000; Deidda et al., 2004, 2006). Estimates of the $c$ parameter obtained for these radar campaigns are displayed in Fig. 8 (bottom) together with the regression line $\mu(I)$ and the interval $c(I)=\mu(I)[1 \pm C V]$ 

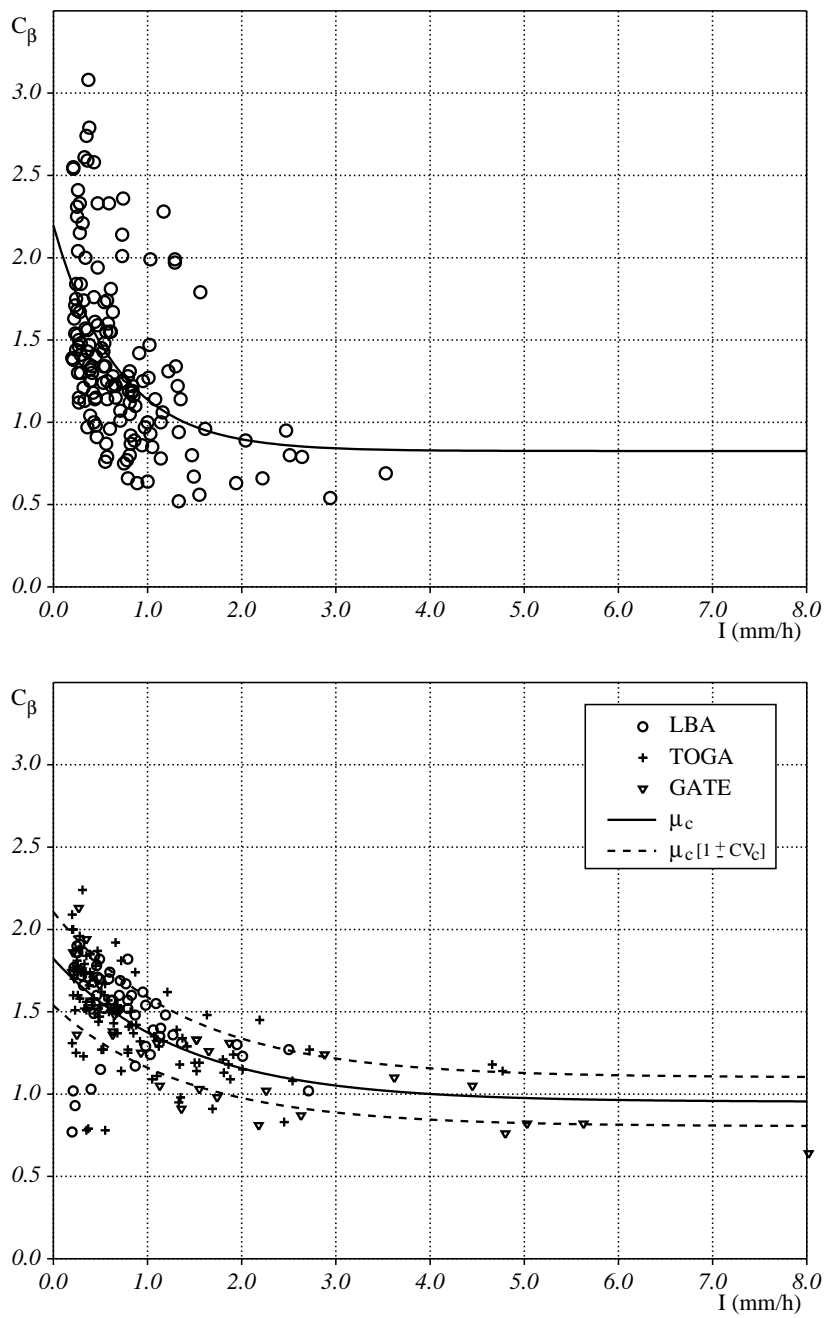

Fig. 8. Sample estimates of the $c$ parameter computed on the 138 selected sequences retrieved over Sardinia are plotted versus mean rainfall intensity $I$ together with the corresponding regression line (top). Sample $c$ estimates for the sequences extracted from TRMM-LBA, TOGA and GATE dataset, corresponding regression lines $\mu(I)$ and confidence limits (bottom).

which was determined by means of the coefficient of variation $C V$. The comparison between Fig. 8 (top) and Fig. 8 (bottom) highlights that $c$ estimates obtained for the sequences extracted in Sardinia from rain gages data differ from the ones obtained from radar data belonging to the campaigns cited above. This outcome might depend on the difference in the analysed data, concerning their type and discretization, as well as on the specific features of the examined region. Independently of the fact that the analysed sequences had similar scale invariant properties, $c$ estimates behaviour does not seem to be interpreted by the same equation for the examined rain gage and radar data. Thus in the following sections we use Eq. (13) which has been calibrated only on Sardinian data (top of Fig. 8).

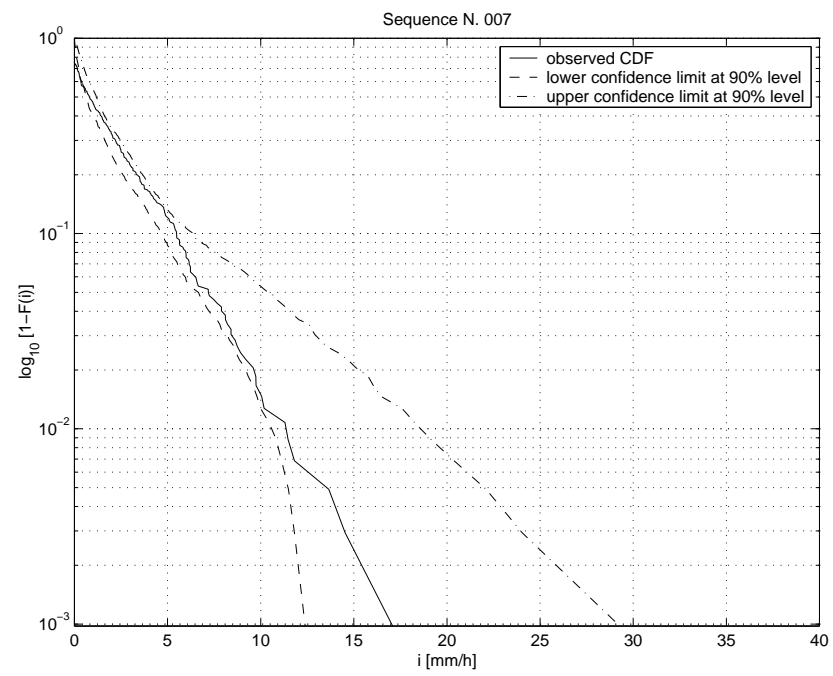

Fig. 9. Cumulative distribution function of rainfall rain rate at higher resolution is compared to the $90 \%$ confidence limits estimated from synthetic fields for one of the analysed sequences. Observed rainfall data have been made spatially homogeneous by means of the modulating function $\bar{\xi}$ in order to be compared to the synthetic ones.

\section{Simulation of synthetic fields}

The effectiveness of the proposed downscaling procedure was evaluated generating a set of 100 synthetic fields for each of the 138 space-time sequences selected in Sect. 5 and then comparing the behaviour of observed and generated rainfall sequences. The STRAIN model was applied for the generation of each set using parameters $\beta=e^{-1}$ and $c$ obtained from Eq. (13) for the same rainfall intensity $I$ of the corresponding observed field. Spatial heterogeneity was later introduced in homogeneous synthetic fields by multiplying rainfall intensity in each cell $(x, y)$ by the same modulating function $\bar{\xi}(x, y)$ given by Eq. (12).

A first comparison was performed among observed and synthetic data within each of the analysed events. Cumulative distribution functions (CDFs) of rainfall intensity obtained at the smallest scales ( $13 \mathrm{~km}$ and $45 \mathrm{~min})$ were computed for each of the observed sequences and compared to the $90 \%$ confidence intervals deduced from the corresponding set of synthetic sequences. In order to merge homogeneous data, for these comparisons, rainfall intensities were previously divided by the modulating function $\bar{\xi}$ while generated fields are not yet multiplied by $\bar{\xi}$. Sample CDFs are within this interval for most of the analysed fields, meaning a correct reproduction of the observed behaviour. As an example the CDF corresponding to one of the selected sequences and its $90 \%$ confidence intervals are displayed in Fig. 9.

A second comparison was performed in order to statistically assess the positive effect induced by the modulating function $\bar{\xi}$. Actually its influence is not apparent from the 


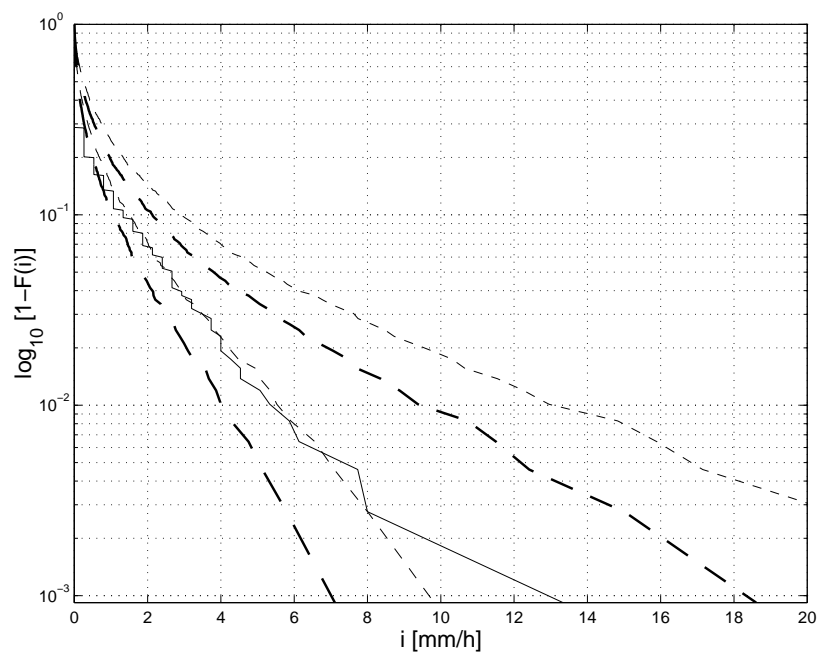

Fig. 10. Cumulative distribution function of rainfall rain rate at 45 min resolution observed over a $13 \mathrm{~km} \times 13 \mathrm{~km}$ grid cell of the A domain (solid line) is compared to the $90 \%$ confidence limits estimated from synthetic fields for the same grid cell. The thick long-dashed lines refer to generations including the $\bar{\xi}$ modulating function while the short-dashed lines are obtained from synthetic homogeneous data (without $\bar{\xi}$ ).

analysis of a single event, due to sample variability of rainfall fields. Thus for each of the grid cells $(13 \mathrm{~km} \times 13 \mathrm{~km})$ of the two selected spatial domains (namely A and B), CDFs of rainfall intensity at time resolution of $45 \mathrm{~min}$ were computed considering all the analysed events retrieved over the corresponding domain. From the synthetic fields generated by the STRAIN model, with and without the superposition of the modulating function, the $90 \%$ confidence intervals in each cell were computed and compared with observed CDF. As an example, in Fig. 10 this comparison is displayed for one of the grid cells belonging to the A domain. The solid line, which represents observed data, is within the thick longdashed lines referring to generation including the modulating function $\bar{\xi}$ while it is partially outside the short-dashed lines representing synthetic homogeneous data (without $\bar{\xi}$ ). The same behaviour was observed for the other grid cells demonstrating the effectiveness of the modulating function in the reproduction of spatial heterogeneity of rainfall mean intensity.

The dependence of the cell mean intensity values on the mean terrain elevation was verified to hold both on the observed events retrieved over the A and B domains (top in Figs. 11 and 12) and on the corresponding simulated sequences (bottom in Figs. 11 and 12).

In Figs. 11 (top) and 12 (top) sample behaviour of the average parameter $\bar{\xi}$ as a function of the terrain elevation is shown for the observed events on the A and B domains. A visual examination of these figures confirms a dependence of $\bar{\xi}$ on the terrain elevation, despite a notable dispersion of the
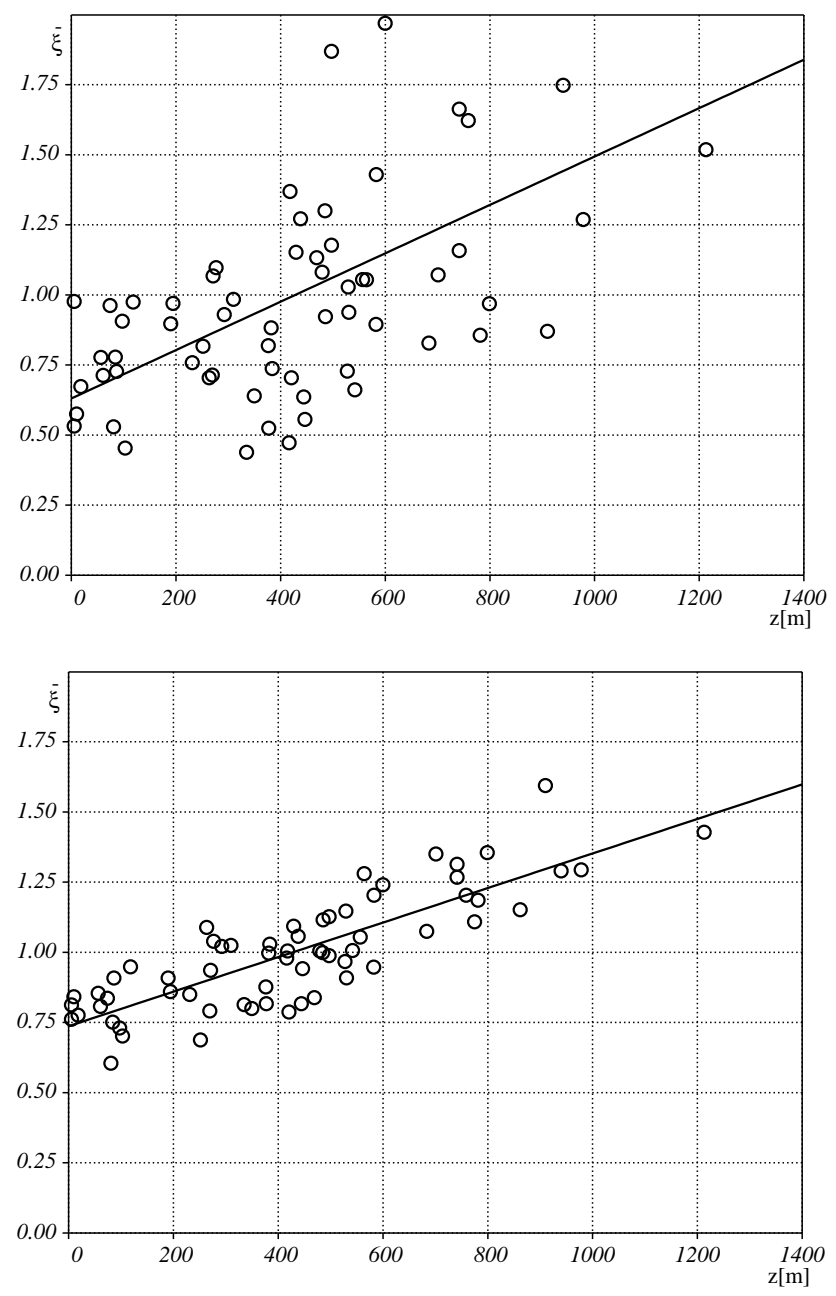

Fig. 11. Averages of the modulating function $\bar{\xi}$ plotted versus mean cell terrain elevation $z$ for the events selected on the A domain. The plots refer to the observed sequences (top) and to one set of synthetic generations (bottom).

data. The regression line slope is $\alpha=0.86 / 1000 \mathrm{~m}^{-1}$ for the A domain and $\alpha=0.40 / 1000 \mathrm{~m}^{-1}$ for the B domain, therefore the slope is, in both cases, different from the one obtained for the entire region analyzing more than 800 events (Sect. 4.2). In order to better understand this apparent discrepancy we remark that the selected sequences are only 70 on the A domain and 68 on the $\mathrm{B}$ domain. The considerable reduction in the number of events with respect to those (about 800) used in the analysis presented in Sect. 4.2 is due to stronger conditions imposed in accepting missing data for the multifractal analysis as discussed at the beginning of Sect. 5. It should be expected that uncertainty in $\alpha$ estimates increases as the number of events used for $\xi$ averaging decreases. As an example the reader can refer to the results of the analysis performed on a regional scale, which are described in Sect. 4.2 and displayed in Fig. 5. Specifically $\alpha$ estimates made on 

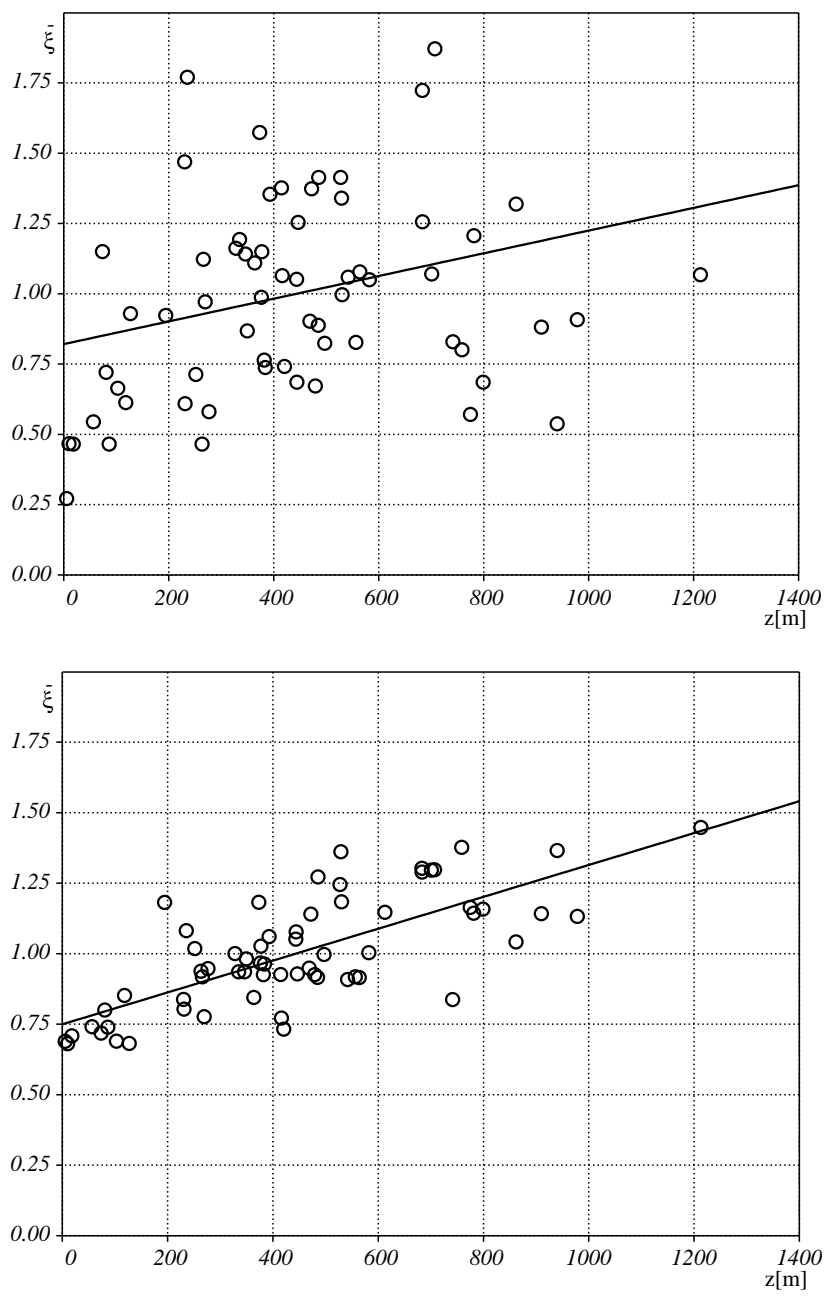

Fig. 12. Averages of the modulating function $\bar{\xi}$ plotted versus mean cell terrain elevation $z$ for the events selected on the B domain. The plots refer to the observed sequences (top) and to one set of synthetic generations (bottom).

sets of 100 events (square symbols in Fig. 5) are very spread along a wide range of values ranging from 0.4 to 0.9 and thus including $\alpha$ estimates obtained for the data belonging to the $\mathrm{A}$ and $\mathrm{B}$ domains. It is therefore difficult to understand whether differences in the two domains, which are superimposed for three quarters, are due to a different local behaviour or to the high variability in $\alpha$ estimates computed on a small group of events. Although our opinion is more favourable to interpret these differences as sample variability, only the availability of longer series of rainfall observation can clarify this issue.

\section{Conclusions}

We investigated the suitability of downscaling procedures for space-time rainfall modelling in case of orographic constraints. With this aim the orographic influence in rainfall fields has been investigated on data retrieved by the high temporal resolution rain gage network of the Sardinian Hydrological Survey. The results have highlighted the existence of spatial heterogeneity in point rain gage measurements as well as in precipitation fields averaged on a regular spatial grid. This heterogeneity was included in the downscaling process by means of a locally defined modulating function which takes into account the different mean values of the precipitation intensity probability distributions depending on terrain elevation.

Due to the high space-time variability in the precipitation events, a great number of events has to be examined in order to retrieve local dependencies in rainfall distributions. The dependency between modulating function and terrain elevation was determined on precipitation data referring to more than 800 events. This relationship was used twice: in order to make the observed sequences spatially homogeneous before performing the scale invariance analysis and then to introduce local orographic constraints in synthetic rainfall fields, which had been generated by means of a homogeneous and isotropic cascade model.

The proposed downscaling procedure is based on the multifractal model STRAIN, with self-similar transformations between space and time scales. The model parsimoniousness allowed to keep only one free parameter depending on the mean rainfall intensity $I$, a quantity that in a forecasting context should be predicted by meteorological models.

The orographic influences highlighted in the analysis are finally introduced multiplying synthetic fields obtained with the STRAIN model by the modulating function previously determined. Results support the feasibility to apply the proposed methodology for the generation of rainfall fields also in case of a mountainous terrain. Despite these encouraging results, orography alone may not completely explain the spatial rainfall heterogeneity features which are also connected to the synoptic situation generating the rainfall events. The feasibility of linking the modulating function to meteorological features of the modelled fields is an important topic for further investigation.

Finally, the way the multifractal analyses were conducted deserve a remark. As a matter of fact, deriving rainfall fields from point measurements may affect the estimates of multifractal exponents and consequently the downscaling model calibration. It would be advisable to understand the effect induced by the use of point measures instead of areal ones. Unfortunately we had no possibility to perform this kind of investigation in the analysed region due to the lack of areal measures. This research issue can be better investigated in orographic regions where both radar data and rain gage measurements are simultaneously available. 
Acknowledgements. The authors are indebted to the anonymous reviewers who provided interesting comments and suggestions to improve our manuscript. We are grateful to Sardinian Hydrological Survey for making data of the high temporal resolution rain gage network available. The research was supported by the Italian Ministry of Education, University and Research (MIUR) - Programma Operativo Nazionale - Misura 1.3, Prot. 13018/2001.

Edited by: L. Ferraris

Reviewed by: two referees

\section{References}

Austin, P. M. and Houze, R. A.: Analysis of structure of precipitation patterns in New England., J. Appl. Meteorol., 11, 926-935, 1972.

Badas, M. G., Deidda, R., and Piga, E.: Orographic influences in rainfall downscaling, Adv. Geosci., 2, 285-292, 2005.

Beven, K. J.: Rainfall-Runoff Modelling: The Primer, Wiley, Chichester, 2001.

Buizza, R., Miller, M., and Palmer, T. N.: Stochastic simulation of model uncertainty in the ECMWF ensemble prediction system, Quart. J. Roy. Meteorol. Soc., 125, 2887-2908, 1999.

Czirok, A., Somfai, E., and Viscek, T.: Experimental evidence for self-affine roughening in a micro-model of geomorphological evolution, Phys. Rev. Lett., 71, 2154-2127, 1993.

Deidda, R.: Rainfall downscaling in a space-time multifractal framework, Water Resour. Res., 36, 1779-1794, 2000.

Deidda, R., Benzi, R., and Siccardi, F.: Multifractal modeling of anomalous scaling laws in rainfall, Water Resour. Res., 35, 1853-1867, 1999.

Deidda, R., Badas, M. G., and Piga, E.: Space-time scaling in highintensity Tropical Ocean Global Atmosphere Coupled OceanAtmosphere Response Experiment TOGA-COARE storms, Water Resour. Res., 40, W02506, doi:10.1029/2003WR002574, 2004.

Deidda, R., Badas, M. G., and Piga, E.: Space-time Multifractality of Remotely Sensed Rainfall Fields, J. Hydrol., doi:10.1016/j.jhydrol.2005.02.036, in press, available online, 2006.

Dubrulle, B.: Intermittency in fully developed turbulence: logPoisson statistics and generalized scale-covariance, Phys. Rev. Lett., 73, 959-962, log-Poisson, 1994.

Ferraris, L., Rudari, R., and Siccardi, F.: The uncertainty in the prediction of flash floods in the northern mediterranean environment, J. Hydrometeorol., 3, 714-727, 2002.

Gupta, V. K. and Waymire, E. C.: A statistical analysis of mesoscale rainfall as a random cascade., J. Appl. Meteorol., 32, 251-267, 1993.

Harris, D., Menabde, M., Seed, A. W., and Austin, G.: Multiscaling characterization of rain fields with a strong orographic influence, J. Geophys. Res., 101(D21), 26 405-26414, 1996.

Jothityangkoon, C., Sivapalan, M., and Viney, N. R.: Tests of a space-time model of daily rainfall in southwest Australia based on nonhomogeneous random cascades, Water Resour. Res., 36, 267-284, 2000.
Kahane, J. P. and Peyriere, J.: Sur certaines martingales de Benoit Mandelbrot, Adv. Math., 22, 131-145, 1976.

Kardar, M., Parisi, G., and Zhang, Y.: Dynamic scaling of growing interfaces, Phys. Rev. Lett., 56, 889-892, 1986.

Lashermes, B., Abry, P., and Chainais, P.: New insights into the estimation of scaling exponents, Int. J. Wavelets, Multir. Inf. Proc., 2, 497-523, 2004.

Lovejoy, S. and Mandelbrot, B. B.: Fractal properties of rain and a fractal model., Tellus, 37A, 209, 1985.

Lovejoy, S. and Schertzer, D.: Generalized scale invariance and fractal models of rain, Water Resour. Res., 21, 1233-1250, 1985.

Mandelbrot, B. B.: Intermittent turbulence in self-similar cascades: divergence of high moments and dimension of the carrier, J. Fluid Mech., 62, 331-358, 1974.

Mandelbrot, B. B.: Negative fractal dimensions an multifractals, Physica A, 163, 306-315, 1990.

Ossiander, M. and Waymire, E. C.: Statistical estimation for multiplicative cascades, Ann. Statist., 28, 1533-1560, 2000.

Ossiander, M. and Waymire, E. C.: On estimation theory for multiplicative cascades, The Indian Journal of Statistics, 64, 323-343, 2002.

Over, T. M. and Gupta, V. K.: A space-time theory of rainfall using random cascades, J. Geophys. Res., 101(D21), 26319-26331, 1996.

Pathirana, A. and Herath, S.: Multifractal modelling and simulation of rain fields exhibiting spatial heterogeneity, Hydrol. Earth Syst. Sci., 6, 659-708, 2002.

Perica, S. and Foufoula-Georgiou, E.: Model for multiscale disaggregation of spatial rainfall based on coupling meteorological and scaling descriptions, J. Geophys. Res., 101, 26 347-26361, 1996.

Purdy, J. C., Harris, D., Austin, G. L., Seed, A. W., and Gray, W.: A case study of orographic rainfall processes incorporating multiscaling characterization techniques, J. Geophys. Res., 106(D8), 7837-7845, 2001.

Schertzer, D. and Lovejoy, S.: Generalized scale invariance in turbulent phenomena, Phys. Chem. Hydrodyn., 6, 623-635, 1985.

Schertzer, D. and Lovejoy, S.: Physical modeling and analysis of rain and clouds by anysotropic scaling of multiplicative processes, J. Geophys. Res., 92(D8), 9693-9714, 1987.

She, Z.-S. and Leveque, E.: Universal scaling laws in fully developed turbulence, Phys. Rev. Lett., 72, 336-339, log-Poisson, 1994.

She, Z.-S. and Waymire, E. C.: Quantized energy cascade and logPoisson statistics in fully developed turbulence, Phys. Rev. Lett., 74, 262-265, log-Poisson, 1995.

Siccardi, F., Boni, G., Ferraris, L., and Rudari, R.: A hydrometeorological approach for probabilistic flood forecast, J. Geophys. Res., 110, D05101, doi:10.1029/2004JD005314, 2005.

Venugopal, V., Foufula-Georgiou, E., and Sapozhnikov, V.: A space-time downscaling model for rainfall, J. Geophys. Res., 104(D16), 19705-19721, 1999a.

Venugopal, V., Foufula-Georgiou, E., and Sapozhnikov, V.: Evidence of dynamic scaling in space-time rainfall, J. Geophys. Res., 104(D24), 31 599-31 610, 1999b. 